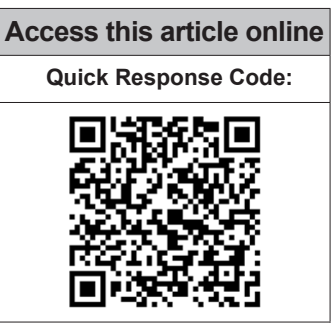

Website:

www.jponline.org

DOI:

10.4103/JLP.JLP_107_18
Departments of Microbiology and ${ }^{2}$ Dermatology and Venereology, All India Institute of Medical Sciences, ${ }^{1}$ Department of Dermatology, STDs and Leprosy, PGIMER and Dr. Ram Manohar Lohia Hospital, New Delhi, India

Address for correspondence: Prof. Seema Sood, Department of Microbiology, All India Institute of Medical Sciences, New Delhi - 110 029, India.

E-mail: seemalsood@ rediffmail.com

Submission: 06-08-2018 Accepted: 26-08-2018

\title{
Typing of Neisseria gonorrhoeae isolates by phenotypic and genotypic techniques in New Delhi, India
}

\author{
Seema Sood, Neeraj Mahajan, Rajendra Singh, Sonu Kumari Agrawal, \\ Trupti Shende, Arti Kapil, Hemanta K. Kar', Vinod K. Sharma²
}

\section{Abstract:}

BACKGROUND: The objective of this study is to investigate gonococcal isolates using phenotypic and genotypic methods.

METHODOLOGY: Sixty gonococcal isolates obtained were examined. Strains were divided into 9 resistant phenotypes: Chromosomally mediated penicillin-resistant Neisseria gonorrhoeae(CMRNGP), penicillinase-producing NG (PPNG), chromosomally mediated tetracycline-resistant NG (CMRNG $\left.{ }^{\top}\right)$, TRNG, PPNG and TRNG, CMRNG ${ }^{P T}$, quinolone resistant NG (QRNG), Azithro R, and decreased susceptibility (DS) to ceftriaxone. These isolates were also subjected to auxotyping and NG-multi-antigen sequence typing (MAST).

RESULTS: Of 60 isolates, 32 (53.33\%) PPNG and only one was CMRNGP; 16 (26.66\%) were $\mathrm{CMRNG}^{\top}$, while $18(30 \%)$ were TRNG. Both PPNG and TRNG found in $13(21.66 \%)$ and none were

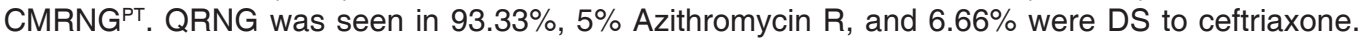
Based on auxotyping, 24 (40\%) nonrequiring, 16 (26.66\%) were proline requiring, $13(21.66 \%)$ arginine requiring while $7(11.66 \%)$ belonged to others. The most common ST was $6058(32.5 \%)$. The discriminatory indices of antibiogram, auxotyping and NG-MAST were $0.77,0.72$, and 0.95 , respectively.

CONCLUSIONS: NG-MAST is the method of choice for epidemiological studies.

Key words:

Antibiogram, auxotyping, Neisseria gonorrhoeae multiantigen sequence typing, Neisseria gonorrhoeae

\section{Introduction}

Conorrhea, caused by Neisseria Jonorrhoeae (NG), is a common sexually transmitted infection in both developing and developed countries. ${ }^{[1]}$ These infections are of concern to healthcare workers as they carry increased risk of morbidity particularly among women as well as neonate. ${ }^{[2]}$ Epidemiological surveillance and strain characterization can provide information regarding the transmission dynamics of this infection, which further influence public health interventions.

This is an open access journal, and articles are distributed under the terms of the Creative Commons Attribution-NonCommercial-ShareAlike 4.0 License, which allows others to remix, tweak, and build upon the work non-commercially, as long as appropriate credit is given and the new creations are licensed under the identical terms.

For reprints contact: reprints@medknow.com
Over the past 40 years, various typing schemes have been devised to characterize the isolates of NG. These have been used to identify the population of strains that circulate in different communities, temporal and geographic changes among the strains, transmission patterns and antibiotic resistance outbreaks. ${ }^{[3,4]}$ Phenotyping and genotyping have been accepted as the two major systems for typing of gonococci. Phenotypic techniques, such as antibiogram, auxotyping and serotyping, have been used in the past either alone or in combination for epidemiological studies and to determine relatedness in NG isolates. However, with new developments in genetics, these are

How to cite this article: Sood S, Mahajan N, Singh R, Agrawal SK, Shende T, Kapil A, et al. Typing of Neisseria gonorrhoeae isolates by phenotypic and genotypic techniques in New Delhi, India. J Lab Physicians 2019;11:45-50. 
now being replaced by molecular techniques. Typing methods can provide information regarding relationship between specific types of gonococci with clinical, diagnostic, and therapeutic problems. ${ }^{[5]}$ In a developing country like India, only few studies have been carried out on typing of NG, and most have focused on phenotypic methods only. ${ }^{[6,7]}$ Therefore, the aim of the present investigation was to analyze the gonococcal isolates in New Delhi by not only phenotypic (antibiogram and auxotyping) methods but also by genotypic methods (NG-multiantigen sequence typing [MAST]). Further, we also examined the association between the typing methods and antimicrobial resistance (AMR). In addition, the ability of different typing methods to differentiate between two isolates has been calculated using the discriminatory index.

\section{Methodology}

\section{Neisseria gonorrhoeae isolates}

A total of 60 consecutive NG isolates collected from patients presenting with cervictis and uretheritis to Dermatology OPD of AIIMS and PGIMER and Dr. R.M.L. Hospital, New Delhi, from July 2010 to June 2012 were studied. Out of 60isolates, 59 were from uretheral swabs of male patients and one from endocervical swab of a female patient. All clinical isolates were preserved in $20 \%$ glycerol broth by deep freezing $\left(-86^{\circ} \mathrm{C}\right.$ ultra-low freezers Freezer, Model 991; Thermo Scientific) at $-70^{\circ} \mathrm{C}$ and also lyophilized using lyophilizer (Free Zone Triad Freeze dry system: Model - 7400030 from Labcono). All 60 isolates were revived on chocolate agar plates at $36^{\circ} \mathrm{C}$ in an atmosphere containing $5 \% \mathrm{CO}_{2}$ before typing.

\section{Antimicrobial susceptibility testing}

Minimum inhibitory concentrations (MICs) of penicillin, tetracycline, ciprofloxacin, spectinomycin, ceftriaxone and azithromycin for all isolates $(n=60)$ was determined by E test method (Biomerieux, France) as per manufacturer's instructions. The results were interpreted as per breakpoint criteria of calibrated dichotomous sensitivity technique. ${ }^{[8]}$ In addition, all isolates were tested for $\beta$-lactamase production by chromogenic cephalosporin method using nitrocefin discs (BD, USA). The WHO NG reference strains F, G, K- P were used for quality control.

The isolates were divided into nine-resistant phenotypes: Chromosomally mediated penicillin-resistant NG $\left(\mathrm{CMRNG}^{\mathrm{P}}\right)\left(\mathrm{CMRNG}^{\mathrm{P}}\right.$ i.e., non-penicillinase producing $\mathrm{NG}$ [PPNG] with penicillin MIC $\geq 2.0 \mu \mathrm{g} / \mathrm{ml}$ and tetracycline MIC $<2.0 \mu \mathrm{g} / \mathrm{ml}$ ), PPNG (PPNG, i.e., $\beta$-lactamase positive with tetracycline MIC $<16 \mu \mathrm{g} / \mathrm{ml}$ ), chromosomally mediated tetracycline resistant NG $\left(\mathrm{CMRNG}^{\mathrm{T}}\right)\left(\mathrm{CMRNG}{ }^{\mathrm{T}}\right.$, i.e., non-PPNG with penicillin $\mathrm{MIC}<2.0 \mu \mathrm{g} / \mathrm{ml}$ and tetracycline MIC $2.0-8.0 \mu \mathrm{g} / \mathrm{ml}$ ),
TRNG (plasmid-mediated tetracycline-resistant NG, i.e., $\beta$-lactamase negative with tetracycline MIC $\geq 16 \mu \mathrm{g} / \mathrm{ml})$, PPNG and TRNG, i.e., $\beta$-lactamase positive with tetracycline MIC $\geq 16 \mu \mathrm{g} / \mathrm{ml}, \mathrm{CMRNG}^{\mathrm{PT}}$, i.e., non-PPNG with penicillin MIC $\geq 2.0 \mu \mathrm{g} / \mathrm{ml}$ and tetracycline MIC 2.0-8.0 $\mu \mathrm{g} / \mathrm{ml}$, quinolone-resistant NG (QRNG) (QRNG, i.e., MIC $\geq 1 \mu \mathrm{g} / \mathrm{ml}$ ), azithromycin resistant (MIC $\geq 1 \mu \mathrm{g} / \mathrm{ml}$ ), and isolates with decreased susceptibility (DS) to ceftriaxone (MIC $0.03-0.25 \mu \mathrm{g} / \mathrm{ml}$ ).

\section{Auxotyping}

Auxotyping was performed as per protocol using gonococcal genetic medium (GGM) $\cdot{ }^{[7]}$ Four different types of media were prepared - one without proline, second without arginine, third containing all seven amino acids, and fourth omitting all 7 amino acids. Standard inoculum size of approximately $3 \times 10^{7} \mathrm{CFU} / \mathrm{ml}$ was used. The medium deficient in substrates was inoculated first, i.e., media lacking all seven amino acids/proline/arginine were inoculated first followed by complete GGM, and finally, the control chocolate agar plates supplemented with yeast extract. The culture plates were then incubated at $37^{\circ} \mathrm{C}$ in $5 \%-10 \% \mathrm{CO}_{2}$ and were examined after 24 and $48 \mathrm{~h}$ of incubation. Isolates with no special nutritional requirement were classified as non-requiring (NR) and those showing absence of growth in proline and arginine deficit media were classified as proline requiring (PR) and arginine requiring (AR), respectively. The WHO NG reference strains $F(A R), K(P R)$ were used for quality control.

\section{Neisseria gonorrhoeae multiantigen sequence typing \\ Isolation of genomic DNA}

Genomic DNA was isolated by boiling method and stored at $-20^{\circ} \mathrm{C}$

All isolates $(n=60)$ were analyzed using NG-MAST as described previously. ${ }^{[9]}$ Both forward and reverse sequences of por and $t b p B$ genes were analyzed by Big Dye Terminator v 3.1 Cycle sequencing kit (Applied Biosystem, California) as per manufacturer's instructions on $3130 \times 1$ genetic analyzer (Applied Biosystems). The forward and reverse sequences of the por and $t b p B$ genes from each isolate were aligned using online available software LALIGN and trimmed to set length from conserved regions as described. Each unique sequence at por and $t b p B$ was assigned an "allelic number" providing each gonococcal isolate with a two-digit allelic profile and each unique allelic profile was designated a numeric ST from the database of the NG-MAST website (http/www.ng-mast.net). The WHO NG reference strains $\mathrm{F}, \mathrm{G}, \mathrm{K}$ - and $P$ were used as control.

\section{Discriminatory index}

The discriminatory capacity of each typing method was determined by calculation of a numerical index, $\mathrm{D}$, 
i.e., the Hunter-Gaston discriminatory index using the following formula: ${ }^{[10]}$

$$
\mathrm{D}=1-\frac{1}{N(N-1)} \sum_{\mathrm{j}=1}^{\mathrm{S}} n_{\mathrm{j}}\left(n_{\mathrm{j}}-1\right)
$$

Where $N$ is the number of isolates in the total population, $\mathrm{S}$ is the total number of types found, and $n_{\mathrm{j}}$ number of isolates belonging to $j^{\text {th }}$ type.

The study was approved by the Ethics Committee, All India Institute of Medical Sciences, New Delhi, India (Ref. No. IEC/NP-A-52/2006 and OP-18/2013 and RP-01/05.08.2013 and IESC/T-170/04.05.2012).

\section{Results}

\section{Antimicrobial susceptibility testing}

Of the 60 isolates, 32 (53.3\%) were PPNG and only 1 (1.6\%) was CMRNG ${ }^{\mathrm{P}}$; 16 (26.6\%) were $\mathrm{CMRNG}^{\mathrm{T}}$, while 18 (30\%) were TRNG. Both PPNG and TRNG were found in $13(21.6 \%)$ while none were $\mathrm{CMRNG}^{\mathrm{PT}}$. QRNG was seen in $93.3 \%$, while $5 \%$ were azithromycin resistant and $6.6 \%$ showed DS to ceftriaxone. All the isolates were susceptible to spectinomycin. $\mathrm{MIC}_{90}$ for penicillin, tetracycline, ciprofloxacin, spectinomycin, ceftriaxone, and azithromycin was $1 \mu \mathrm{g} / \mathrm{ml}, 2 \mu \mathrm{g} / \mathrm{ml}, 3 \mu \mathrm{g} / \mathrm{ml}, 5 \mu \mathrm{g} / \mathrm{ml}, 0.012 \mu \mathrm{g} / \mathrm{ml}$, and $0.25 \mu \mathrm{g} / \mathrm{ml}$, respectively [Table 1]. There were no multidrug-resistant (MDR)-NG and extensively drug-resistant (XDR)-NG as per recently proposed criteria. Further, isolates showing DS to ceftriaxone were resistant to penicillin, ciprofloxacin and less susceptible to tetracycline [Table 2]. One isolate was also resistant to azithromycin. Antibiogram had a discriminatory index of 0.77 .

\section{Auxotypes}

Out of 60 isolates, $24(40 \%)$ were NR, $16(26.6 \%)$ were PR, 13 (21.6\%) AR, and 7 (11.6\%) belonged to others. The discriminatory index of auxotyping was 0.72 .

Genotyping (Neisseria gonorrhoeae-multiantigen sequence typing)

Sixty gonococcal isolates were distributed among 40 different STs, $29(72.5 \%)$ of which were novel. The majority of STs $(77.5 \%$, 31 of 40$)$ were represented by a single isolate and remaining STs included between 2 and 13 isolates. The most common ST was $6058(n=13)$ followed by ST621, ST1572, ST1751, ST2990, ST6069, ST9774, ST9783, and ST9786 (two each). A total of 31 por and $27 t b p B$ alleles were found in this study. The discriminatory index of NG MAST was 0.95.

\section{Association between minimum inhibitory} concentrations and auxotypes

All auxotypes were sensitive to spectinomycin. Isolates exhibiting resistance to azithromycin belonged to auxotypes NR, PR, AR - one each and DS to ceftriaxone belonged to auxotypes $\mathrm{NR}(n=1), \operatorname{PR}(n=2)$, AR $(n=1)$. MIC ${ }_{90}$ for ceftriaxone was highest in auxotype NR $(0.012 \mu \mathrm{g} / \mathrm{ml})$ followed by $\operatorname{AR}(0.008 \mu \mathrm{g} / \mathrm{ml})$ and PR $(0.006 \mu \mathrm{g} / \mathrm{ml})$. However, there was no difference in $\mathrm{MIC}_{90}$ for azirthromycin $(0.25 \mu \mathrm{g} / \mathrm{ml})$ in these three auxotypes.

Association between m i n i m $\mathrm{m}$ inhibitory concentrations and Neisseria gonorrhoeae-multi-antigen sequence typing

Isolates showing DS to ceftriaxone belonged to ST6069 $(n=2)$, ST8156, ST6083 (one each) and the ones resistant to azithromycin belonged to ST9779, ST1572,

Table 1: Antimicrobial susceptibility of Neisseria gonorrhoeae isolates $(n=60)$ by $E$-test

\begin{tabular}{lcccc}
\hline Antibiotic & Susceptible $(\%)$ & Less susceptible/decreased susceptibility $(\%)$ & Resistant $(\%)^{\text {MIC }_{90}(\mu \mathrm{g} / \mathrm{ml})}$ \\
\hline Penicillin & 0 & $27(45)$ & $33(55)^{*}$ & 1 \\
Tetracycline & $9(15)$ & $17(28.3)$ & $34(56.6)^{\#}$ & 2 \\
Ciprofloxacin & $2(3.3)$ & $2(3.3)$ & $56(93.3)^{\# \#}$ & 3 \\
Spectinomycin & $60(100)$ & 0 & 0 & 5 \\
Ceftriaxone & $56(93.3)$ & $4(6.7)$ & 0 & 0.012 \\
Azithromycin & $57(95)$ & 0 & $3(5)$ & 0.25 \\
\hline
\end{tabular}

${ }^{*}$ PPNG - 32 (53.3\%), " ${ }^{\text {TTNG }}-18(30 \%),{ }^{*} \mathrm{HLR}-30(50 \%) . \mathrm{PPNG}=$ Penicillinase producing $N$. gonorrhoeae, TRNG = Tetracycline-resistant $N$. gonorrhoeae, $\mathrm{HLR}=$ High level resistant, MIC = Minimum inhibitory concentration, N. gonorrhoeae = Neisseria gonorrhoeae

Table 2: Susceptibility pattern of ceftriaxone decreased susceptible strains to other antimicrobial agents

\begin{tabular}{lcclc|c|c|cccc}
\hline \multicolumn{2}{c}{ Ceftriaxone } & \multicolumn{2}{c}{ Penicillin } & \multicolumn{2}{c}{ Tetracycline } & \multicolumn{2}{c}{ Ciprofloxacin } & \multicolumn{2}{c}{ Azithromycin } \\
\hline MIC & Category & MIC & Category & MIC & Category & MIC & Category & MIC & Category & \\
\hline 0.064 & DS & 1.0 & CMR & 4.0 & CMR & 12 & HLR & 0.5 & S & - \\
0.064 & DS & 1.5 & CMR & 0.75 & LS & 8 & HLR & 0.125 & S & - \\
0.064 & DS & $>32$ & R & 0.5 & LS & 1.5 & R & 0.125 & S & + \\
0.125 & DS & 1.5 & CMR & 1 & LS & 1 & R & 1 & R & - \\
\hline
\end{tabular}

MIC = Minimum inhibitory concentration, DS = Decreased susceptibility, R = Resistant, LS = Less susceptible, HLR = High-level resistant, CMR = Chromosomally mediated resistance, $S=$ Susceptible,$+=\beta$ lactamase producer, $-=\beta$ lactamase Non-producer 
and ST6083 (one each). Out of 40 identified STs, the most common ST was $6058(n=13)$. Out of 13 ST6058, $12(92.3 \%)$ were PPNG and none were CMRNG $; 9$ (69.2\%) were $\mathrm{CMRNG}^{\mathrm{T}}$ while 4 (30.7\%) were TRNG. Both PPNG and TRNG were found in 4 (30.76\%). All isolates belonging to ST6058 were resistance to ciprofloxacin and sensitive to azithromycin, ceftriaxone and spectinomycin. There was significant association between ST6058 and PPNG $(P=0.00)$ and $\mathrm{CMRNG}^{\mathrm{T}}(P=0.003)$.In all other resistance phenotypes, no association was found.

\section{Discussion}

Besides characterization of gonococcal strains, typing is also useful in studying of relationship between NG isolates during outbreaks in sexual networks, investigating transmission of antibiotic-resistant strains, and differentiating re-infection from treatment failure. Typing methods used should be discriminatory; however, at the same time, it is important that they are easy to perform, practical, and inexpensive. Among the various typing methods to characterize NG isolates i.e., phenotypic and genotypic, the widely used phenotypic methods are based on auxotyping, serotyping, and antimicrobial susceptibility testing. The phenotypic methods are less efficient and do not meet the desired discriminatory index. ${ }^{[11]}$ Further, auxotyping being laborious and time-consuming, has been replaced by genotypic methods world over. Among genotypic methods, NG-MAST is a leading method which is based on sequencing and allows international comparison of data and tracing of gonococci of public health importance. In India, very few studies have been carried out on gonoccocal typing methods and most have focused on phenotypic methods only. ${ }^{[6,7]}$ No study from India comparing the phenotypic and genotypic methods has been documented so far to the best of our knowledge.

As regards AMR pattern as a typing method, many studies from India only differentiate isolates into PPNG and non-PPNG or into TRNG and non-TRNG. The incidence of PPNG varies from $8 \%$ to $88.9 \%$ in various parts of India. ${ }^{[12]}$ In the present study, 53.3\% strains were PPNG while only $1.6 \%$ demonstrated chromosomal resistance, thereby implying that majority of penicillin resistance is due to $\beta$-lactamase production. The $\beta$-lactamases in this organism are TEM-1 type. Mutations in TEM gene leading to an ESBL phenotype have been reported in the past, particularly in Enterobacteriaceae. ${ }^{[13]}$ Gonococci have a plastic genome and therefore, we need to be vigilant and keep a close watch on the evolution of TEM gene in our isolates. The PPNG rates in Japan, US, and Australia have been reported to be $1.2 \%, 42.2 \%$ and $11.6 \%$ respectively. ${ }^{[14-16]}$ Further, we observed that $30 \%$ of gonococcal isolates were TRNG while $26.6 \%$ exhibited chromosomal resistance to tetracycline.
A study from India showed increasing trends of TRNG from $28.1 \%$ to $58.5 \%$ between year 2009 and 2011. ${ }^{[12]}$ However, reporting for tetracycline in India is technique based and not all laboratories categorize the non-TRNG strains further. ${ }^{[12]}$ A recent study from Sanghai reports tetracycline resistance rates to be $54.7 \%$ of which TRNG was $31 \%$ and chromosomal resistance was seen in $23.7 \%$, a finding similar to ours. ${ }^{[17]}$ Overall, we observed both, chromosomal and plasmid-mediated resistance to penicillin and tetracycline. In the present study, $93.3 \%$ isolates were resistant to ciprofloxacin. Although no longer recommended for treatment of gonorrhea, ciprofloxacin resistance rates were frightening. Previously, very high rates of resistance to ciprofloxacin have been reported from Delhi. On reviewing literature, we found that considerable differences exist in ciprofloxacin resistance from different parts of India ranging from $49.1 \%$ to $100 \% .{ }^{[12]}$ All over the world, there is a variation in the rates of resistance to fluoroquinolones and is seen in $10.2 \%$ to $27.3 \%$ cases in US, 33.6\% in Australia and 78.2\% in Japan. ${ }^{[15,16,18]}$ Rates of resistance to drug used in syndromic management in our country were low. A total of $6.6 \%$ isolates exhibited DS to ceftriaxone while $5 \%$ were azithromycin resistant. There appears no threat to the in-use therapies administered in syndromic approach, i.e., cefixime $400 \mathrm{mg}$ plus azithromycin $1 \mathrm{~g}$ orally as a single dose. Isolates with DS to ceftriaxone almost always exhibit resistance to quinolones or quinolones and penicillin as observed in our study too. Overall, chromosomal resistance was more often associated with DS to ceftriaxone [Table 2]. Our findings, although on a small number of isolates, are in line with the national as well as international data. ${ }^{[19-23]}$ Our isolates were susceptible to spectinomycin which support the finding of the previous studies from India, where no resistance to this drug has been documented except isolated case report. ${ }^{[24]}$ There were no MDR-NG and XDR-NG.

The discriminatory index of antibiogram in our study was 0.77 and was higher than previous studies reported from India. ${ }^{[11,25]}$ This could possibly be because we have taken into account both, plasmid as well as chromosomal resistance to antimicrobials. The NR and PR auxotypes are most common auxotypes in Delhi and Mumbai and the same was observed by us. ${ }^{[11]}$ Restriction of certain auxotypes to different geographical areas can help in global epidemiological studies. Both PPNG and TRNG were demonstrating NR auxotype as the most common auxotype; however, the association was not significant. This is in line with the previous Indian studies. ${ }^{[11]}$ Isolates exhibiting resistant to azithromycin and DS to ceftriaxone belonged to NR, PR, and AR auxotypes. The discriminatory index of auxotyping was 0.72 , which is higher than previous studies reported from India. ${ }^{[11,25]}$ This is surprising as we, in our study, 
have not investigated the less common nutritional requirements such as methionine, hypoxanthine, uracil, thiamine, pyrophosphate, and isoleucine. However, some workers in India have combined auxotyping with another phenotypic method to achieve a desirable discriminatory index. ${ }^{[11]}$

NG-MAST has been used as a tool to enhance surveillance of gonococcal AMR and to study the clonal spread in high-risk population. Our strains were distributed among 40 different STs of which $29(72.5 \%)$ were novel. This reflects the adaptation of gonococci to this geographic regionand evolution of STs in India. The most common ST was ST6058 the same has been reported by us in the previous year. ${ }^{[26]}$ ST6058 was significantly associated with resistance phenotypes, PPNG and CMRNG ${ }^{\mathrm{T}}$. In all other resistance phenotypes, no association was found. All isolates belonging to ST6058 were sensitive to drugs in use. Further, the DS isolates belonged to different STs (ST6069 [ $n=2]$ ], ST8156 and ST6063 [one each]), thereby demonstrating heterogenicity which suggests the introduction of strains from multiple sources rather than clonal spread. None of our DS isolates belonged to ST 1407 , the internationally documented MDR gonococcal clone. ${ }^{[27,28]}$ Isolates exhibiting DS to ceftriaxone belonged to auxotypes NR, AR (one each), PR $(n=2)$, and the NR and PR auxotypes surprisingly belonged to the same ST6069 and had similar por and tbp B alleles. Therefore, most probably, these isolates represent infection with same strain. Isolates resistant to azithromycin belonged to auxotypes NR, PR, and AR (one each), of which the AR strain belonged to a novel ST9779.

The $D$ value of NG-MAST in our study was 0.95 , thereby implying that it is a highly efficient technique. Further, molecular epidemiological approaches help to furnish additional information on the origin and spread of AMR.

\section{Conclusions}

Gonococci are notorious for high rates and rapid spread of drug-resistant strains and therefore warrant that such facilities be introduced in the routine surveillance programs to achieve better disease control. However, their prohibitive cost preclude use in a setting like ours where it is feasible only when funds are available for such research activities. On the other hand, although phenotypic methods have several limitations, they allow the comparison of recent data with the older data.

\section{Acknowledgments}

We are thankful to Dr. Manju Bala Professor and Consultant Microbiologist, Regional STD Teaching, Training and Research Center and Coordinator, WHO GASP SEAR, RRL, VMMC and SJH, New Delhi, India, for providing $\mathrm{WHO}$ reference strains.

\section{Financial support and sponsorship}

This work was financially supported by the ICMR, Government of India [grant no. IRIS-ID No. 2006-0685E]; and the All India Institute of Medical Sciences [project no.F.5-59/IRG/2010/RS].

\section{Conflicts of interest}

There are no conflicts of interest.

\section{References}

1. Van Looveren M, Ison CA, Ieven M, Vandamme P, Martin IM, Vermeulen $\mathrm{K}$, et al. Evaluation of the discriminatory power of typing methods for Neisseria gonorrhoeae. J Clin Microbiol 1999;37:2183-8.

2. Moodley P, Martin IM, Ison CA, Sturm AW. Typing of Neisseria gonorrhoeae reveals rapid reinfection in rural South Africa. J Clin Microbiol 2002;40:4567-70.

3. Palomares JC, Lozano MC, Perea EJ. Antibiotic resistance, plasmid profile, auxotypes and serovars of Neisseria gonorrhoeae strains isolated in Sevilla (Spain). Genitourin Med 1990;66:87-90.

4. Pierce RL, Thomas JC, Sparling PF, Fisher W, Davis RH, Alcorn T, et al. An epidemiological evaluation of the use of microbiological tools for identifying gonorrhoea infection networks. Int J STD AIDS 1999;10:316-23.

5. Divekar AA, Gogate AS, Shivkar LK. Association between auxotypes, serogroups, and antibiotic susceptibilities of Neisseria gonorrhoeae isolated from women in Mumbai (Formerly Bombay), India. Sex Transm Dis 1999;26:358-63.

6. Agarwal SK, Deb M, Prakash K, Sharma AK. Auxotyping of Neisseria gonorrhoeae as an additional epidemiological marker. Indian J Med Res 1992;95:227-9.

7. La Scolea LJ Jr., Young FE. Development of a defined minimal medium for the growth of Neisseria gonorrhoeae. Appl Microbiol 1974;28:70-6.

8. Bell SM, Pham JN, Carter IW, Hanrahan JK. Antibiotic Susceptibility Testing by the CDS Method. A Manual for Medical and Veterinary Laboratories. $6^{\text {th }}$ ed. Randwick, Australia: The Prince of Wales Hospital, South Eastern Area Laboratory Services; 2011. p. 62.

9. Martin IM, Ison CA, Aanensen DM, Fenton KA, Spratt BG. Rapid sequence-based identification of gonococcal transmission clusters in a large metropolitan area. J Infect Dis 2004;189:1497-505.

10. Hunter PR, Gaston MA. Numerical index of the discriminatory ability of typing systems: An application of Simpson's index of diversity. J Clin Microbiol 1988;26:2465-6.

11. Khaki P, Bhalla P, Sharma P, Chawla R, Bhalla K. Epidemilogical analysis of Neisseria gonorrhoeae isolates by antimicrobial susceptibility testing, auxotyping and serotyping. Indian J Med Microbiol 2007;25:225-9.

12. Bala M, Kakran M, Singh V, Sood S, Ramesh V, Members of WHO GASP SEAR Network, et al. Monitoring antimicrobial resistance in Neisseria gonorrhoeae in selected countries of the WHO South-East Asia region between 2009 and 2012: A retrospective analysis. Sex Transm Infect 2013;89 Suppl 4:iv28-35.

13. Rawat D, Nair D. Extended-spectrum $\beta$-lactamases in gram negative bacteria. J Glob Infect Dis 2010;2:263-74.

14. Ohnishi M, Ono E, Shimuta K, Watanabe H, Okamura N. Identification of TEM-135 beta-lactamase in penicillinase-producing Neisseria gonorrhoeae strains in Japan. Antimicrob Agents Chemother 2010;54:3021-3.

15. Kirkcaldy RD, Harvey A, Papp JR, Del Rio C, Soge OO, Holmes KK, et al. Neisseria gonorrhoeae antimicrobial susceptibility surveillance-The gonococcal isolate surveillance project, 27 sites, 
United States, 2014. MMWR Surveill Summ 2016;65:1-9.

16. Lahra MM, Enriquez RP; National Neisseria Network. Australian gonococcal surveillance programme, 1 July to 30 September 2015. Commun Dis Intell Q Rep 2016;40:E179-81.

17. Trecker MA, Waldner C, Jolly A, Liao M, Gu W, Dillon JA, et al. Behavioral and socioeconomic risk factors associated with probable resistance to ceftriaxone and resistance to penicillin and tetracycline in Neisseria gonorrhoeae in Shanghai. PLoS One 2014;9:e89458.

18. Shimuta K, Unemo M, Nakayama S, Morita-Ishihara T, Dorin M, Kawahata T, et al. Antimicrobial resistance and molecular typing of Neisseria gonorrhoeae isolates in Kyoto and Osaka, Japan, 2010 to 2012: Intensified surveillance after identification of the first strain (H041) with high-level ceftriaxone resistance. Antimicrob Agents Chemother 2013;57:5225-32.

19. Bala M, Ray K, Gupta SM, Muralidhar S, Jain RK. Changing trends of antimicrobial susceptibility patterns of Neisseria gonorrhoeae in India and the emergence of ceftriaxone less susceptible N. gonorrhoeae strains. J Antimicrob Chemother 2007;60:582-6.

20. Tapsall JW. Annual report of the Australian gonococcal surveillance programme, 2005. Commun Dis Intell 2006;30:205-10.

21. Akasaka S, Muratani T, Yamada Y, Inatomi H, Takahashi K, Matsumoto $\mathrm{T}$, et al. Emergence of cephem- and aztreonam-high-resistant Neisseria gonorrhoeae that does not produce beta-lactamase. J Infect Chemother 2001;7:49-50.

22. Muratani T, Akasaka S, Kobayashi T, Yamada Y, Inatomi H,
Takahashi K, et al. Outbreak of cefozopran (penicillin, oral cephems, and aztreonam)-resistant Neisseria gonorrhoeae in Japan. Antimicrob Agents Chemother 2001;45:3603-6.

23. Ito M, Deguchi T, Mizutani KS, Yasuda M, Yokoi S, Ito S, et al. Emergence and spread of Neisseria gonorrhoeae clinical isolates harboring mosaic-like structure of penicillin-binding protein 2 in central Japan. Antimicrob Agents Chemother 2005;49:137-43.

24. Bala M, Ray K, Salhan S. First case of spectinomycin resistant Neisseria gonorrhoeae isolate in New Delhi, India. Sex Transm Infect 2005;81:186-7.

25. Kulkarni S, Bala M, Risbud A. Antimicrobial susceptibility testing, auxotyping, and serotyping of Neisseria gonorrhoeae strains isolated in India. Sex Transm Dis 2012;39:188-90.

26. Mahajan N, Sood S, Singh R, Kapil A, Das BK, Sreenivas V, et al. Antimicrobial resistance and Neisseria gonorrhoeae multiantigen sequence typing profile of Neisseria gonorrhoeae in New Delhi, India. Sex Transm Dis 2016;43:506-16.

27. Ota KV, Ng LK, Melano RG, Martin IE, Brown EM, Richardson SE, et al. Identification of sexual networks through molecular typing of quinolone-resistant Neisseria gonorrhoeae in Ontario, Canada. Sex Transm Dis 2011;38:811-4.

28. Tapsall JW, Ray S, Limnios A. Characteristics and population dynamics of mosaic penA allele-containing Neisseria gonorrhoeae isolates collected in Sydney, Australia, in 2007-2008. Antimicrob Agents Chemother 2010;54:554-6. 